\title{
APLICACIÓN INDUSTRIA 4.0 EN LOGÍSTICA DE RAPPI “ZONA T”, DE BOGOTÁ D.C., COLOMBIA
}

\author{
Industry 4.0 application on RAPPI logistics in "Zona T", in Bogotá D.C., Colombia \\ Martha Lucia Belmonte Rodríguez ', Rodrigo Eduardo Pardo Calderón², Luis Felipe Mogollón Loaiza \\ ${ }^{1-3}$ Corporación Universitaria UNITEC, Escuela de Ingeniería, capítulo IISE 617, Colombia. \\ Email:172181524@unitec.edu.co, 272171501@unitec.edu.co,372201506@unitec.edu.co
}

(Recibido marzo 09 y aceptado junio 02 de 2021)

\section{Resumen}

El presente manuscrito documenta la investigación realizada a la empresa de paquetería y mensajería RAPPI de la ciudad Bogotá y, cómo esta aplica la Industria 4.0 en su modelo logístico. En la cual se usan los conocimientos adquiridos en áreas de ingeniería, métodos y logística para el planteamiento de posibles mejoras al proceso de entregas Delivery. El área de estudio fue la Zona T de la ciudad de Bogotá ubicada entre las calles 79 a 85 y las carreras 11 a 15, debido a que es una de las zonas de mayor importancia gastronómica y comercial, con un alto flujo de "Rappitenderos". Para el análisis logístico, se realizó estudio de campo y la utilización de fuentes bibliográficas como artículos emitidos por periódicos y revistas locales, así como, estudios de Universidades Nacionales sobre el impacto de la industria 4.0 en el empleo y plataformas virtuales SoyRappi-App para repartidores, Waze y Google Maps. Con esta investigación, se busca analizar el impacto de las tecnologías 4.0 en este tipo empresas de economía colaborativa, promoviendo la eficiencia en el proceso de entregas y servicio al cliente.

Palabras clave: entrega, industria 4.0, logística, plataforma digital.

\begin{abstract}
This document corresponds to the research carried out at the RAPPI enterprise based on parcel and courier company located in Bogotá city and, how Industry 4.0 applies in its logistics model. The knowledge acquired in the engineering, methods and logistics areas is used to propose possible improvements to the delivery process. The study area was Zone T of the Bogotá city located between streets 79 to 85 and Avenues 11 to 15, because it is one of the areas of greatest gastronomic and commercial relevance, with a high flow of "SoyRappi" in much more of this. For the logistic analysis, a field study was carried out and the use of bibliographic sources such as local articles issued by newspapers and magazines, studies from National Universities based on the impact of Industry 4.0 on employment and virtual platforms like SoyRappiApp for deliverymen, Waze and Google Maps. This research seeks to analyze the impact of 4.0 technologies in this kind of collaborative economy companies promoting efficiency in the delivery process and customer service.
\end{abstract}

Key words: delivery, industry 4.0, logistics, digital platform.

\section{INTRODUCCIÓN}

En el Foro Económico Mundial realizado del 25 al 29 de enero de 2021, líderes del mundo, ONGs, académicos y científicos concluyeron que la "COVID-19 aceleró el despliegue de las nuevas tecnologías hacia la Cuarta Revolución Industrial" y que el uso de la tecnología será un aspecto fundamental para la recuperación económica, ya que se ha logrado que muchas de las actividades que se realizaban de manera presencial ahora se efectúen 
de forma eficiente en la virtualidad [1]. Este tipo de tecnologías como los sistemas logísticos interconectados, el Internet de las cosas, el Big Data y la realidad aumentada se han aplicado en diferentes sectores como el Delivery, el Retail, la moda y la industria hacia al control de procesos, calidad, eficiencia operativa y mejores experiencias al cliente. A continuación, se mencionan algunos de los considerados pilares de la tecnología 4.0:

- Computación en la nube: Servicios de gestión de recursos que se ofrecen a través de internet y que se cobran por lo que se consume [2].

- Internet de las cosas: Conjunto de tecnologías que permiten la comunicación entre objetos físicos y máquinas a través de la internet para generar nuevos modelos de negocios e ingresos, eficiencia operativa y mejores experiencias al cliente [3].

- Comunicación M2M: Cualquier tecnología que permita que dos dispositivos se comuniquen y envíen datos entre sí como el RFID, Bluetooth y la Wifi. Este tipo de conexión permite la optimización de los procesos de forma automática [4].

- Big Data y Analítica: Técnicas de analítica avanzada de grandes conjuntos de datos que facilita la toma de decisiones mediante el análisis de información que antes era inaccesible o inutilizable [5].

En Colombia, sectores como el cervecero y de logística, han sido algunos de los más destacados en la implementación de tecnologías para una operación más controlada; "Bavaria ha optado por la digitalización de sus plantas en el país para hacer más eficiente el proceso de producción de la cerveza" [6] y Servientrega "desarrolló una plataforma web autoservicio para agilizar procesos y proporcionar información en línea a los usuarios que les permita controlar la operación de su negocio" [7].

La logística en cualquier sector es fundamental ya que es un proceso sujeto a variabilidad en tiempos, calidad y precios que afecta, principalmente, la percepción del cliente final en cuanto al servicio recibido. Es así, que las empresas que se han reinventado y han logrado que sus procesos sean más flexibles y se acoplen a tecnologías como la inteligencia artificial, la analítica de datos y las plataformas virtuales han logrado que las variabilidades antes mencionadas disminuyan en gran medida.

Empresas como Rappi, Domicilios.com y iFood que hacen parte de la actividad del Delivery, han reinventado esta operación a través del uso de las plataformas virtuales que permiten que el usuario final adquiera sus productos sin desplazarse y que tenga conocimiento sobre la hora de entrega. En este orden de ideas, Rappi, por ejemplo, llegó a ser muy flexible, porque pasó de prestar un servicio de solo pedidos de restaurantes y supermercados, a la gestión y entrega de electrodomésticos, cosmetología, floristería, deportes y compra de cualquier tipo de producto a través de sus servicios exclusivos [8].

Con base a la premisa anterior y la importancia de la tecnología 4.0 en la logística, el presente manuscrito tiene como finalidad, analizar la empresa Rappi y su modelo de trabajo en la zona $\mathrm{T}$, sector de gran afluencia gastronómica, con el fin de identificar las tecnologías aplicadas a la entrega de pedidos y proponer alternativas de mejora en su operación.

\section{ANTECEDENTES}

Rappi es una multinacional fundada en Bogotá-Colombia, en el año 2015 por 3 jóvenes: Simón Borrero, fundador; Sebastián Mejía y Felipe Villamarín, cofundadores, quienes trabajaron en la creación de esta empresa tecnológica que funciona a través de una aplicación gratuita, descargable para los sistemas Android y iOS [9]. "Su objetivo es facilitar a las personas el acceso a diferentes formas de realizar sus compras o envíos locales sin necesidad de moverse del lugar donde se encuentran" [10] quienes expandieron esta iniciativa por diferentes países de América Latina como México, Brasil, Uruguay y Argentina a través de la App. A continuación, se menciona en la Tabla 1, los servicios disponibles en su plataforma para Colombia [11]: 
Tabla 1. Servicios ofrecidos por Rappi para Colombia.

\begin{tabular}{cl}
\hline Tipo de servicio & \multicolumn{1}{c}{ Productos y servicios ofrecidos } \\
\hline Lo que quieras & Cajero automático a Domicilio, se puede retirar desde \$20.000 hasta \$400.000. \\
\hline Lo que sea o Rappi favor & $\begin{array}{l}\text { Comprar algo y entregarlo como un postre, ropa, calzado, accesorios o recoger algo y } \\
\text { entregarlo. }\end{array}$ \\
\hline Rappi travel & Servicios de vuelos (Avianca, Latam, Viva Air, Satena, Easy fly) \\
\hline Vapeadores vype & Se enfoca en todos los insumos que se encuentran alrededor de los vapeadores. \\
\hline Vida sana & $\begin{array}{l}\text { Se encuentran los diferentes fruver, almacenes de cadena y tiendas especializadas en } \\
\text { productos verdes. }\end{array}$ \\
\hline Mercado solidario & $\begin{array}{l}\text { Los usuarios/consumidores podrán donar menús de restaurantes aliados a la plataforma } \\
\text { Rappi desde \$7.500 y serán entregados a las fundaciones inscritas. }\end{array}$ \\
\hline Mi tienda & $\begin{array}{l}\text { Las empresas pueden ofrecer sus productos de e-commerce, alimentos empaquetados o } \\
\text { menús. }\end{array}$ \\
\hline Life miles y Davipuntos & \begin{tabular}{l} 
Tienen la opción de convertir millas o Davipuntos en rappicréditos para comprar en Rappi. \\
\hline Shoping
\end{tabular} \\
\hline El usuario podrá acceder a comprar cursos online de Excel, idiomas, lectura etc. \\
\hline $\begin{array}{l}\text { Compra de tecnología, moda, belleza, hogar, regalos etc. También se pueden adquirir } \\
\text { productos 100\% colombianos. }\end{array}$ \\
\hline
\end{tabular}

\section{MODELO DE TRABAJO}

El modelo de trabajo y operación, se gestiona a través de su plataforma digital donde Rappi conecta empresas y clientes con el canal de distribución (Rappitendero) con el fin de que el usuario tenga la posibilidad de acceder a un sinnúmero de productos y servicios sin necesidad de desplazarse. De esta manera, las empresas tienen la posibilidad de ampliar su demanda a través de la modalidad de domicilio sin incurrir en costos de entrega. En este modelo, interactúan 3 actores: las empresas aliadas que ofrecen sus productos y servicios, los usuarios-clientes que acceden a ellos dependiendo su ubicación y cobertura y, los Rappitenderos quienes se encargan de llevar el pedido como forma de obtener ingresos complementarios a otras actividades, de forma flexible y no como una jornada laboral estricta.

Dentro del modelo, la aplicación informa cuando ocurren las siguientes etapas [12]:

- El Rappitendero toma el pedido.

- El restaurante empieza a preparar.

- Se despacha el pedido, es decir se entrega.
- Rappitendero.

- Cuando ocurren novedades en la entrega.

- Ruta del Rappitendero hasta la casa.

Los elementos involucrados dentro de este modelo son:

- Plataformas digitales: Son el medio a través del cual interactúan las empresas, usuarios-clientes y Rappitenderos para la compra, venta y aceptación del pedido de productos y servicios, basada en la economía de mercados.

- Empresas aliadas: Son las organizaciones que se inscriben para ofrecer sus productos y servicios a través de la plataforma digital y quienes a su vez monitorean la ubicación de los Rappitenderos y su situación a fin de informar cualquier detalle al usuario.

- Usuario-cliente: Persona que se registra como usuario para hacer uso de los servicios que ofrece la plataforma digital.

- Rappitendero: Persona encargada de entregar el producto o realizar el servicio solicitado por el usuario, quien no maneja contrato directo con la empresa que administra la aplicación. Para trabajar 
en determinadas zonas deben contar con un número de puntos que se obtienen por la calificación del servicio y las entregas realizadas al día.

- $\quad$ Rappi Pit Stops: Son puntos físicos adaptados para cubrir ciertas necesidades de los Rappitenderos como parqueo de bicicletas, acceso a baños, espacio para descanso y espera de pedidos. Estas zonas tienen una capacidad de 30 a 50 personas y el horario de funcionamiento es de 6:00 am a 10:00 pm todos los días [13].

En cuanto a los horarios, aunque los colaboradores Rappi, no se encuentran sujetos a cumplir una jornada laboral, son libres de trabajar los días y en los horarios que prefieran; pueden ganar entre $\$ 9.000$ y $\$ 14.000$ pesos por hora, inclusive $\$ 120.000$ pesos en 12 horas. Sin embargo, para quienes utilizan esta modalidad como único trabajo, deben hacer mínimo 8 horas diarias para ganar al mes $\$ 832.000$ pesos, así también, deben tener cuidado al rechazar pedidos o estar mucho tiempo inactivos ya que pueden ser sancionados [14].

\section{METODOLOGIA}

Para el planteamiento de la propuesta se realizó lo siguiente:

- Investigación sobre la Industria 4.0, así como de las tecnologías que hacen parte y su aplicación en sectores industriales como el de Delivery.

- Selección y caracterización de la empresa de Delivery y su modelo de negocio.

- Identificación y delimitación de la zona de estudio (estado de vías, obras en curso).

- Caracterización comercial de la zona e identificación de los puntos Rappi en los establecimientos de comida.

- Caracterización del proceso logístico desde la solicitud del producto hasta la entrega al usuario final y análisis de las tecnologías aplicadas.

- Conclusiones y recomendaciones.

\section{RESULTADOS}

La zona T es una zona delimitada por dos calles peatonales que forman una $T$ entre las calles 79 a 85 y las carreras 11 a 15 (Ver. Figura1) donde predominan los núcleos residenciales y de servicios que convierten al sector en uno de los epicentros comerciales y de servicios más importantes del norte de la ciudad [15].
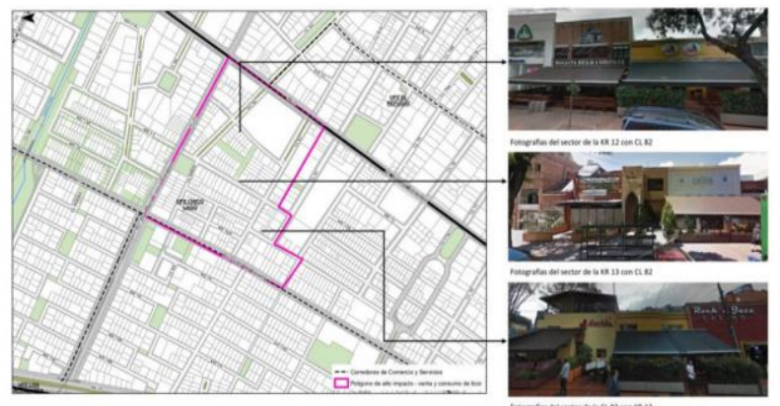

Fuente: SDP Dirección de Norma Urbana, 2019

Figura 1. Mapa de la zona T [16].

Para el 2019, la localidad comenzó con la construcción y adecuación de 73 mil metros cuadrados de espacio público como adecuación de zonas verdes y nivelación de los pasos peatonales [17] por ser una zona de gran dificultad en movilidad especialmente en horas pico. Actualmente, estas obras se encuentran finalizadas. La zona T presenta en su mayoría, establecimientos dedicados al entretenimiento y la gastronomía. Se encuentran centros comerciales muy importantes como el Andino y el Jumbo de la 80 y restaurantes reconocidos de comida internacional y nacional.

En la visita realizada, se identificaron aproximadamente 110 establecimientos gastronómicos de los cuales 89 son aliados Rappi entre ellos Bogotá Beer Company, Presto, Macdonalds, WOK, Di Lucca, entre otros, donde la mayor parte de los Rappitenderos se ubican frente al establecimiento ocupando andenes y obstruyendo el paso. Para un domiciliario es más rentable hacer pedidos dentro de esta zona ya que el valor del domicilio es más alto. 
A su vez, se identificó que el único Rappi Pit Stops ubicado en City parking Andino, cra 11 \# 84, resulta insuficiente para todos los Rappitenderos, sumando la crisis sanitaria actual, se hace necesario que Rappi habilite al menos otro punto para su permanencia y así se disminuya la aglomeración en la zona. Por esta misma causa, muchos Rappitenderos han pensado en cambiar de empresa en búsqueda de mayores posibilidades de trabajo, porque este sector Rappi tiene mucha oferta de domiciliarios, lo cual dificulta el acceso a la prestación del servicio.

A continuación, se describen las tecnologías 4.0 utilizadas y, en la Figura 2, cómo funciona la aplicación:

- Computación en la nube e internet de las cosas: Rappi diseñó una plataforma web donde se administra y controla el proceso de pedidos, la cual puede ser usada ingresando a través de una URL o mediante la descarga de la aplicación para sistemas iOS y Android. Adicionalmente, esta empresa hace uso de redes sociales sobre los servicios ofrecidos como estrategia de marketing.

- Comunicación M2M: La empresa genera códigos ID que permiten la identificación de los Rappitenderos, así como hacer uso de la aplicación. Se usa la geolocalización para que los usuarios puedan ver el recorrido del Rappitendero y recibir notificaciones a través de un chat sobre el estado del pedido.

- Big Data y Analítica: A través de la aplicación, las empresas pueden monitorear cuantos pedidos han despachado, hacer seguimiento a las ventas e indicadores operativos. Rappi utiliza AppsFlyer la cual "permite a desarrolladores de apps, anunciantes y agencias medir y optimizar estrategias destinadas a la captación de clientes a través del uso de algoritmos y la integración con cientos de Ad Networks" 10 [18]; siendo pionera mundial en atribución móvil al encontrarse en el $98 \%$ de los aparatos inteligentes de todo el mundo [19].

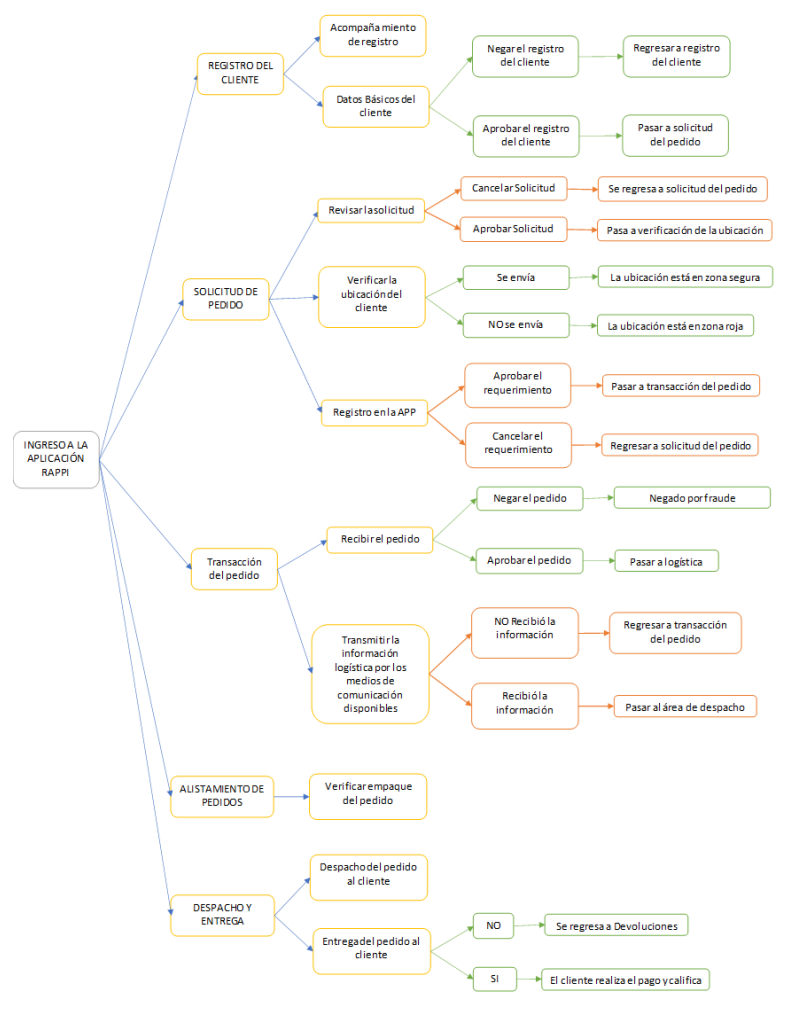

Figura 2. Proceso logístico Rappi.

\section{CONCLUSIONES}

- En la actualidad, Rappi se ha posicionado como una de las plataformas más importantes, ya que ha alcanzado altos niveles tecnológicos y de marketing; este negocio va más allá de gestionar pedidos de comidas, porque apunta a satisfacer todo tipo de necesidades de los usuarios.

- A partir del cambio al que tuvo que ajustarse todas las empresas con la crisis causada por la Covid-19, fue necesario la flexibilización de sus modelos de negocio, de tal manera que, sus estrategias de crecimiento se adaptaran e hicieran uso de las nuevas tecnologías para optimizar sus operaciones y manejar eficientemente la información de sus usuarios.

- Las empresas de Delivery como Rappi han demostrado que es posible desarrollar servicios que permitan un mejor control de la cadena de 
distribución con la aplicación de las tecnologías 4.0, esta aplicación permite disminuir costos operativos para las empresas aliadas, al conectar a través de una aplicación a los clientes con una red de domiciliarios y a estos con los comercios.

\section{RECOMENDACIONES}

- Se sugiere para disminuir el número de Rappitenderos en la zona T, se ubique un Rappi Pit Stop en la torre de la 78- Cr 12 \# 78-40, punto estratégico por la cercanía con los puntos de comidas y el espacio suficiente para su instalación.

- Uno de los retos de Rappi, es lograr diferenciarse más de los competidores como ifood, que han comenzado una etapa de mayor inversión en tecnología para mejorar la experiencia del usuario.

- La calidad de vida que se le puede brindar a una persona es vital dentro de las organizaciones, por lo anterior, se recomienda que Rappi mejore sus condiciones laborales, que velen por la seguridad del domiciliario y brinden algún tipo de seguro contra accidentes.

\section{REFERENCIAS}

[1] B. Rohde, (8 de 02 de 2021). Tendencias de la Industria 4.0 en 2021. Obtenido de Cuatro Cero: https://cuatro-cero.mx/noticias/tendencias-de-laindustria-4-0-en-2021/

[2] Hewlett Packard. (2021). Computación en la nube. Obtenido de https://www.hpe.com/es/es/what-is/ cloud-computing.html

[3] SAP. (2021). Internet de las cosas. Recuperado el 28 de 03 de 2021, de https://www.sap.com/ latinamerica/insights/internet-of-things.html

[4] Atria innovation. (19 de 11 de 2019). Comunicación M2M o Machine to Machine, en qué consiste. Recuperado el 28 de 03 de 2021, de Atria innovation: https://www.atriainnovation.com/comunicacionm2m-que-es/

[5] IBM. (2021). Analítica de Big Data. Recuperado el 28 de 03 de 2021, de https://www.ibm.com/analytics/ es/es/hadoop/big-data-analytics/

[6] Furore. (11 de 06 de 2019). 3 Sectores de la industria en Colombia que están invirtiendo en tecnología 4.0. Obtenido de Furore: 3 Sectores de la industria en Colombia que están invirtiendo en tecnología 4.0

[7] Revista Semana. (28 de 10 de 2020). Logística 4.0, un salto para el sector en Colombia. Obtenido de Semana: https://www.semana.com/contenidoseditoriales/transporte/articulo/logistica-40-unsalto-para-el-sector-en-colombia/202000/

[8] Rappi. (22 de 04 de 2021). Rappi Página Oficial. Obtenido de https://www.rappi.com/jobs/how-werecruit

[9] Rappi. (13 de 01 de 2018). Cómo nació Rappi explicado por su fundador Simón Borrero. Obtenido de Rappi: https://blog.rappi.com/como-naciorappi/

[10] L. O. Silva, Y. C. Blanco \& J. Livingston, (s.f.). Comunicación, Capitalismo y Crítica en la Colombia Digital. Obtenido de Universidad de la Costa: https://repositorio.cuc.edu.co/ bitstream/handle/11323/6149/Domicilios\%2c\%20 Aplicaciones\%20y\%20Econom\%c3\%ada\%20 colaborativa.\%20El\%20caso\%20de\%20Rappi. pdf?sequence $=1$ \&isAllowed $=y$

[11] Rappi. (22 de 04 de 2021). Rappi Página Oficial. Obtenido de https://www.rappi.com/jobs/how-werecruit

[12] Rappi. (3 de 08 de 2016). Qué es Rappi y cómo funciona: conoce cómo mejoramos tu calidad de vida. Obtenido de Rappi: https://blog.rappi.com/ que-es-rappi/

[13] Rappi. (22 de 04 de 2021). Rappi Página Oficial. Obtenido de https://blog.soyrappi.com/pit-stops/

[14] K. Patiño, (5 de 07 de 2019). Tras quema de maletas, ¿por qué se quejan los Rappitenderos? El Tiempo. Recuperado el 04 de 22 de 2021, de El Tiempo: https://www.eltiempo.com/tecnosfera/ apps/quejas-de-rappitenderos-y-respuestas-derappi-284776

[15- Alcaldía Mayor de Bogotá. (11 de 2020). 16] Documento Técnico de Soporte del Plan de 
Ordenamiento Territorial. Obtenido de http:// www.sdp.gov.co/sites/default/files/CONCEJO/4DOCUMENTO_TECNICO_DE_SOPORTE_CONCEJO/ DT11_Anexo01_Poligonos_de_alto_impacto.pdf

[17] Canal Capital. (25 de 06 de 2020). Avanza obra de construcción en la Zona Rosa de Bogotá. Obtenido de: https://conexioncapital.co/avanza-obra-deconstruccion-en-la-zona-rosa-de-bogota/

[18] Merkle. (2 de 09 de 2020). AppsFlyer, saca el máximo rendimiento a tus campañas de Mobile. Obtenido de https://www.merkleinc.com/es/es/ blog/appsflyer-campanas-mobile

[19] I. Briceño, (23 de mayo de 2019). Radio Santa fe Bogotá. Obtenido de Radio Santa fe Bogotá: http:// www.radiosantafe.com/2019/05/23/tecnologiaappsflyer-de-rappi-ha-permitido-crecimiento-dela-compania/ 